\title{
Machinelike or Humanlike? A Literature Review of Anthropomorphism in AI-Enabled Technology
}

\author{
Mengjun Li \\ City University of Hong Kong \\ mengjunli3-c@my.cityu.edu.hk
}

\author{
Ayoung Suh \\ Sungkyunkwan University \\ aysuh@skku.edu
}

\begin{abstract}
Due to the recent proliferation of AI-enabled technology (AIET), the concept of anthropomorphism, human likeness in technology, has increasingly attracted researchers' attention. Researchers have examined how anthropomorphism influences users' perception, adoption, and continued use of AIET. However, researchers have yet to agree on how to conceptualize and operationalize anthropomorphism in AIET, which has resulted in inconsistent findings. A comprehensive understanding is thus needed of the current state of research on anthropomorphism in AIET contexts. To conduct an in-depth analysis of the literature on anthropomorphism, we reviewed 35 empirical studies focusing on conceptualizing and operationalizing AIET anthropomorphism, and its antecedents and consequences. Based on our analysis, we discuss potential research gaps and offer directions for future research.
\end{abstract}

\section{Introduction}

In recent years, artificial intelligence (AI) has become a popular and important topic among individuals, organizations, and societies $[1,2]$. Key examples of AI-enabled technology (AIET) include chatbots, virtual agents, autonomous vehicles, smart speakers, and social robots. These are increasingly used in diverse domains, such as customer service [3], healthcare [4], agriculture [5], education [6], and retail [7]. According to Fortune Business Insights [8], the global AI market was valued at USD 20.67 billion in 2018 and is expected to reach USD 202.57 billion by 2026. Experts predict that AIET will become omnipresent in all areas of human life within the next few years $[1,2]$.

This technology's AI capabilities (e.g., machine learning, natural language, and reasoning processing) $[9,10]$ enable humanlike functions. These capabilities make autonomous vehicles operate autonomously, chatbots communicate with humans naturally, and social robots exhibit emotional responses [9, 11], thereby allowing AIET to interact with humans in humanlike ways $[9,12]$. Researchers thus argue that the humanlike characteristics of AIET are unique technological features that can influence users' perception, adoption, and continued use of this technology [12-16].

Researchers have employed the concept of anthropomorphism to capture human likeness of AIET - a term that refers to the attribution of humanlike characteristics to nonhumans. Studies using the concept of anthropomorphism have confirmed that technologies' human likeness influences how users interact with specific technologies $[12,14]$. Scholars have highlighted that anthropomorphism mitigates individuals' anxiety and stress when interacting with unfamiliar virtual agents and satisfies their social needs $[13,17]$.

Despite the increasing scholarly attention to this concept, research on anthropomorphism in AIET contexts is fragmented in terms of definitions and operationalizations. Given the importance of this issue to information systems (IS) and related disciplines, a literature review is needed to integrate previous studies of anthropomorphism in AIET in order to obtain an overview of the current state of research, identify gaps, and indicate potential avenues for future research.

This study had three objectives. First, we aimed to systematically review previous studies to identify patterns, including research trends, theoretical foundations, and methodologies. Second, we aimed to analyze emergent themes and organize them systematically, according to, among other aspects, conceptual and operational definitions of anthropomorphism. Third, based on the gaps identified in the literature, we aimed to suggest possible directions for future research.

This paper is organized as follows: Section 2 introduces the research background by defining the concept of AIET and anthropomorphism in AIET contexts. Section 3 details the procedures that we followed in our literature search. Section 4 and 5 provides an analysis of the 35 identified studies and presents the current state of research. Section 6 
discusses the study's implications, limitations, and the scope for further research.

\section{Research background}

\subsection{AIET}

AI enables existing and new types of information technology to perform tasks intelligently $[2,10]$ based on specific technological capabilities, such as machine learning, deep learning, text analysis, natural language processing, and speech recognition [1, 10]. According to Rzepka and Berger [10], AIET comprises two types of technology. The first is technology that uses AI capabilities to enhance existing technologies' functions (i.e., AI-enhanced technology). The second is technology that uses AI capabilities to develop new products (i.e., AI-based technology). Recent research has shown that AIET has strong implications at the individual, organization, and societal levels $[1,10]$. The adoption of, use of, and interaction with these technologies have thus become common and important research topics in many fields, including IS [14], psychology [17], and marketing [18].

\subsection{Anthropomorphism}

Thanks to its AI capabilities, AIET exhibits humanlike traits, such as displaying humor or emotions [12], showing the courtesy [19], and expressing empathy [20] when interacting with humans. In the AIET context, the phenomenon of attributing humanlike traits to these technologies is called anthropomorphism [9, 19]. Recently, anthropomorphism has been identified as an important factor influencing the perception, adoption, and continued use of AIET [12, 15, 16, 21]. For instance, Waytz et al. [21] found that anthropomorphism promoted trust in autonomous vehicles. Moussawi et al. [12] showed that anthropomorphism enhanced individuals' perceived enjoyment when they interacted with smart speakers, which led to an increase in adoption intention. Likewise, Wagner et al. [16] reported that anthropomorphism increased smart speakers' likeability, thereby promoting the intention to adopt them.

Conversely, other studies have found that anthropomorphism has a negative effect on individuals' willingness to use AIET [3, 14, 22]. For example, $\mathrm{Lu}$ et al. [22] argued that anthropomorphism catalyzes a sense of threat to human identity, undermining people's willingness to adopt service robots. Similarly, Gursoy et al. [14] and Lin et al. [3] posited that anthropomorphism triggers negative emotions, resulting in objections to the use of AIET.

Given the inconsistent findings on the effects of anthropomorphism on the perception and adoption of AIET, researchers have had difficulty consolidating the existing knowledge. Therefore, a systematic literature review is needed to synthesize the existing findings and understand the current state of anthropomorphism research.

\section{Literature search and identification}

Following the guidelines of Webster and Watson [23], we took a two-stage approach to searching for and identifying articles on anthropomorphism in AIET. Figure 1 shows the literature search and identification procedures implemented. In the first stage, we conducted a systematic search in several online databases, including Web of Science, EBSCOhost, ProQuest, SAGE, ScienceDirect, Taylor and Francis Online, and Scopus, to find relevant articles. At this stage, we decided to target only peerreviewed journal articles included in the Social Sciences Citation Index to ensure the reviewed articles' quality. Keywords, such as "anthropomorphism," "artificial intelligence technology," and "AI-enabled technology," were used to conduct the literature search. To ensure that the most relevant articles were included in the results, we conducted a manual search of 11 major IS journals and seven conference proceedings (Figure 1). The search yielded 819 relevant articles. After removing duplicates, 457 articles remained.

In the second stage, we applied a set of inclusion and exclusion criteria to confirm the articles' relevance and appropriateness for the analysis. Although anthropomorphism in technologies attracted attention before 2000 , in this study, we aim to focus on the more recent research on AIET and anthropomorphism. Therefore, we searched papers published between 2000 and 2020. The inclusion criteria were articles that (1) were published during the period 2000-2020, (2) principally focused on anthropomorphism, and (3) included conceptual and/or operational definitions of anthropomorphism. According to the exclusion criteria, we rejected studies that (1) examined anthropomorphism in non-AIET contexts and/or (2) did not present empirical results. Based on these criteria, 35 articles were identified as relevant. A forward and backward search of these articles' references yielded no additional studies. The final sample for analysis thus consisted of 35 articles. The reviewed articles are listed in the Online Supplementary Material. 


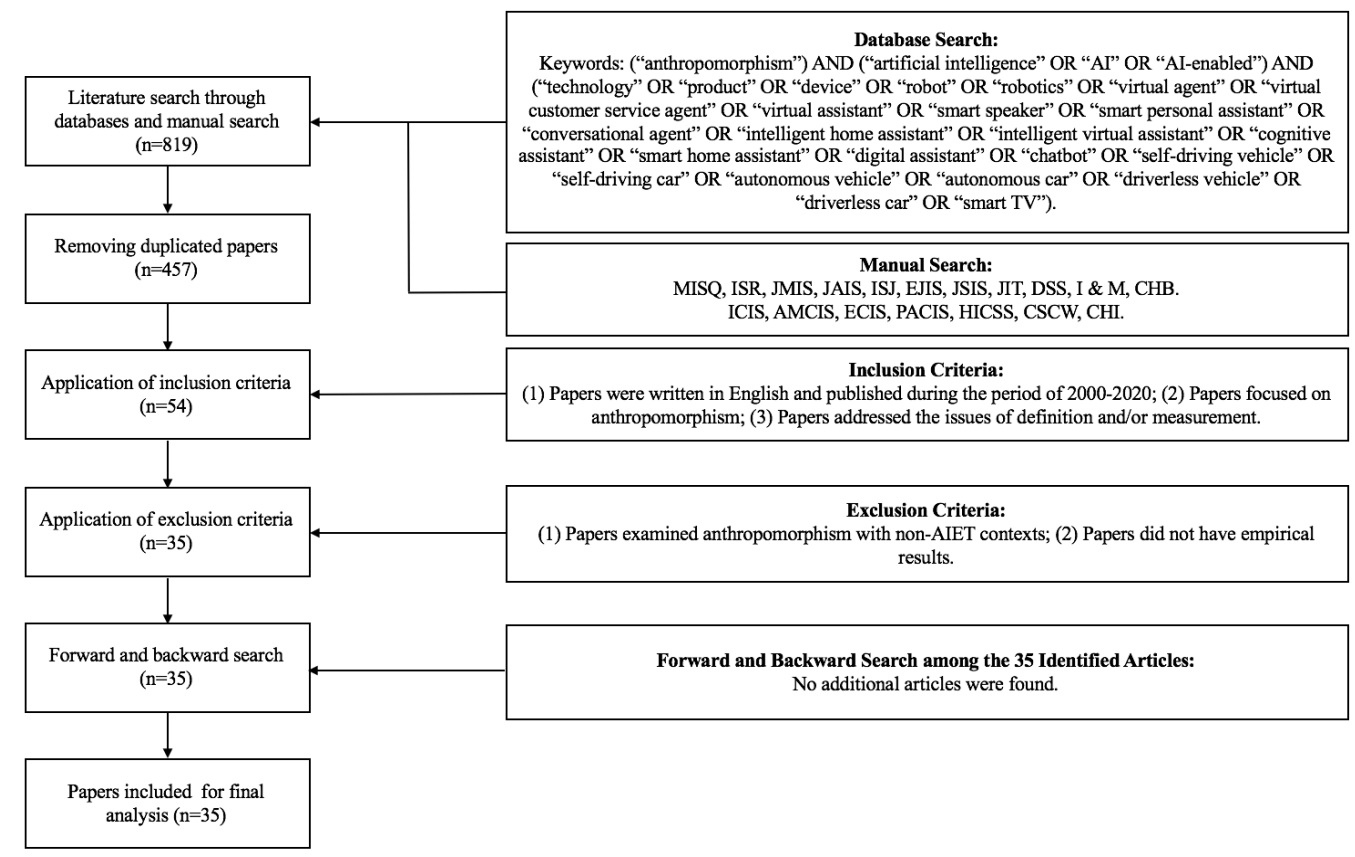

Figure 1. Literature search and identification procedures

\section{Overview}

Following Webster and Watson [23], to analyze the research trends, technologies, theoretical foundations, research methods, and contexts of AIET research, the first author coded and the co-authors cross-checked the results. In case of disagreement, they discussed the coded results until they reached a consensus.

\subsection{Overview of research trends}

First, we examined the 35 papers' bibliometric data. As shown in Figure 2, the number of studies on anthropomorphism in AIET contexts has grown since 2018. Four-fifths of the included studies $(n=28)$ were published between 2019 and 2020. This recent increase in publications can be attributed to the rapid development of AI, suggesting that anthropomorphism is an important research topic attracting more researchers' interest. Moreover, $29 \%$ of the identified articles $(\mathrm{n}=10)$ were published in early 2020, which indicates that a significant increase in relevant research can be expected in the following years.

Three main research streams emerged from the reviewed articles. The first stream pays particular attention to the ways in which anthropomorphism affects users' perceptions of AIET. For example, Waytz et al. [21] explored the effect of anthropomorphism on individuals' overall trust in autonomous vehicles. Banks [24] examined the influence of anthropomorphism on users' perceptions of morality and dependability of performing tasks on smart speakers and chatbots. Kim et al. [17] assessed how anthropomorphism contributes to different levels of perception of smart speakers' competence and warmth.

The second stream of research focuses on the effect of anthropomorphism on the intention to adopt AIET. For instance, Rzepka et al. [25] analyzed the effect of anthropomorphism on the intention to use smart speakers for shopping from a cost-benefit perspective. Moussawi et al. [12] explored how anthropomorphism influenced the perceived enjoyment and initial trust in smart speakers, which in turn affected the intention to adopt AIET. Melián-González et al. [26] examined how anthropomorphism shaped individuals' intentions to adopt chatbots.

The third stream of research emphasizes the influence of anthropomorphism on AIET service evaluations. For example, Qiu et al. [27] investigated the effect of anthropomorphism on evaluations of service experiences that include interacting with social robots. Choi et al. [28] studied how anthropomorphism influenced the relationship between language styles of AIET and service encounter evaluations. Toader et al. [29] explored the indirect effect of anthropomorphism on individuals' service encounter evaluations via their trust in chatbots. 


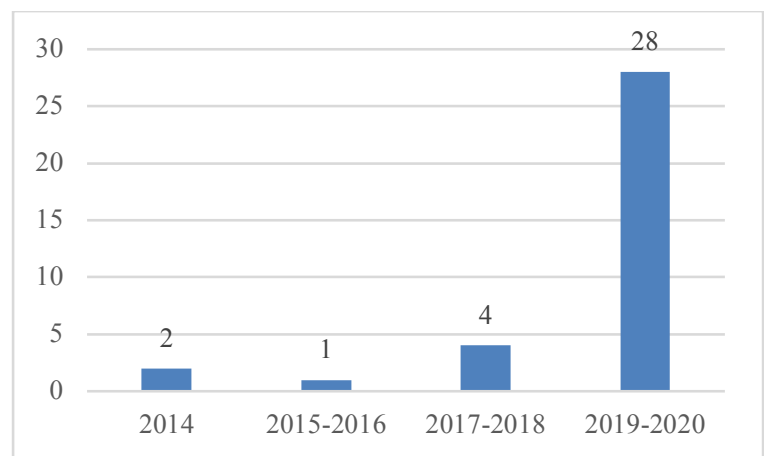

Figure 2. The publication years of the articles

\subsection{Overview of technologies}

The analyzed articles cover various types of technology. Over a third $(37 \% ; n=13)$ of the studies focused on chatbots, such as Cleverbot and Facebook Messenger bots. More than one-fourth $(29 \% ; n=10)$ examined smart speakers, with most studies using Amazon Alexa and Siri. Seventeen percent $(n=6)$ focused on social robots, such as Nadine and Hilton's Connie. Around $11 \%(\mathrm{n}=4)$ dealt with autonomous vehicles, mostly investigating autonomous driving systems embedded in driving simulators. About $6 \%$ (n $=2$ ) explored expert systems, such as AlphaGo and financial robo-advisors as automated investment advisory services. Finally, about $6 \%(\mathrm{n}=2)$ used general terms such as AI devices [14] and AI machines [30].

\subsection{Overview of theoretical foundations}

Table 1 presents the theories and conceptual frameworks adopted by studies on anthropomorphism. We found that $69 \%(n=24)$ of the studies based their empirical investigations on a theoretical foundation, whereas $31 \%(\mathrm{n}=11)$ did not incorporate any theoretical backgrounds in their research model.

Social response theory $[31,32]$ as the most popular theoretical foundation, was used to examine how anthropomorphism elicits social responses from AIET users [20, 33-35]. Uncanny valley theory [19, 36, 37] was used to explore how anthropomorphism affects individuals' emotional responses to AIET. Other studies adopted trust theory [21, 38, 39], social presence theory [40], the computers are social actors (CASA) paradigm [17, 29], and the modality-agencyinteractivity-navigability (MAIN) model [41] to investigate how anthropomorphism affects perceptions of AIET. The unified theory of acceptance and use of technology (UTAUT) [16, 22, 26], technology acceptance model (TAM) [12, 42], artificially intelligent device use acceptance (AIDUA) model [3], and cognition-motivation-emotion framework [14] were used to understand the effects of anthropomorphism on technology adoption. The theory of reasoned action (TRA) was used to examine the relationship between anthropomorphism and people's AIET usage behaviors [25].

Table 1. Summary of theoretical foundations

\begin{tabular}{|c|c|c|}
\hline Theory & Description & $\begin{array}{l}\text { Refe } \\
\text { renc } \\
\text { es }\end{array}$ \\
\hline $\begin{array}{l}\text { Social } \\
\text { response } \\
\text { theory }\end{array}$ & $\begin{array}{l}\text { Social response theory holds that people } \\
\text { view computer systems as social actors } \\
\text { and respond to them socially. }\end{array}$ & $\begin{array}{l}{[20,} \\
33- \\
35]\end{array}$ \\
\hline $\begin{array}{l}\text { Uncanny } \\
\text { valley theory }\end{array}$ & $\begin{array}{l}\text { Uncanny valley theory suggests that } \\
\text { individuals first show positive an affinity } \\
\text { toward humanlike technologies; } \\
\text { however, when they become too } \\
\text { humanlike, people experience a feeling } \\
\text { of eeriness or uncanniness. }\end{array}$ & $\begin{array}{l}{[19,} \\
36, \\
37]\end{array}$ \\
\hline UTAUT & $\begin{array}{l}\text { UTAUT is a framework that explores } \\
\text { factors influencing individuals' } \\
\text { technology adoption and usage behavior. }\end{array}$ & $\begin{array}{l}{[16,} \\
22, \\
26]\end{array}$ \\
\hline TAM & $\begin{array}{l}\text { The model posits that perceived ease of } \\
\text { use and perceived usefulness are two } \\
\text { primary factors influencing individuals' } \\
\text { intentions to accept and use a technology }\end{array}$ & $\begin{array}{l}{[12,} \\
42]\end{array}$ \\
\hline $\begin{array}{l}\text { Cognition- } \\
\text { motivation- } \\
\text { emotion } \\
\text { framework }\end{array}$ & $\begin{array}{l}\text { It proposes that during a decision- } \\
\text { making process, people go through } \\
\text { several stages of cognitive appraisals and } \\
\text { creating emotions toward the stimulus } \\
\text { that leads to behavioral intentions. }\end{array}$ & [14] \\
\hline $\begin{array}{l}\text { AIDUA } \\
\text { model }\end{array}$ & $\begin{array}{l}\text { It explains how individuals utilize a } \\
\text { multi-step process to determine their } \\
\text { adoption intentions of AIET. }\end{array}$ & [3] \\
\hline Trust theory & $\begin{array}{l}\text { It states that a humanlike machine would } \\
\text { increase individuals' trust when the } \\
\text { machine performs intelligently and } \\
\text { competently. }\end{array}$ & $\begin{array}{l}{[21,} \\
38 \\
39]\end{array}$ \\
\hline $\begin{array}{l}\text { Social } \\
\text { presence } \\
\text { theory }\end{array}$ & $\begin{array}{l}\text { It argues that individuals believe that } \\
\text { they are interacting with a genuine social } \\
\text { actor and their interactions are socially } \\
\text { meaningful. }\end{array}$ & [40] \\
\hline $\begin{array}{l}\text { CASA } \\
\text { paradigm }\end{array}$ & $\begin{array}{l}\text { It assumes that people mindlessly apply } \\
\text { social heuristics to computers. }\end{array}$ & $\begin{array}{l}{[17,} \\
29]\end{array}$ \\
\hline MAIN model & $\begin{array}{l}\text { It explores ways in which four } \\
\text { technological features (i.e., modality, } \\
\text { agency, interactivity, and navigability) } \\
\text { shape user perceptions. }\end{array}$ & [41] \\
\hline TRA & $\begin{array}{l}\text { It describes the link among individuals' } \\
\text { attitudes, intentions, and behaviors. }\end{array}$ & {$[25]$} \\
\hline $\begin{array}{l}\text { Extended-self } \\
\text { theory }\end{array}$ & $\begin{array}{l}\text { It postulates that individuals see certain } \\
\text { other people, as well as certain tangible } \\
\text { and intangible possessions as part of } \\
\text { themselves. }\end{array}$ & [18] \\
\hline $\begin{array}{l}\text { Three-factor } \\
\text { theory of } \\
\text { anthropomorp } \\
\text { hism }\end{array}$ & $\begin{array}{l}\text { It focuses on three psychological } \\
\text { determinants (i.e., elicited agent } \\
\text { knowledge, effectance motivation, and } \\
\text { sociality motivation) to explain when } \\
\text { people are likely to anthropomorphize } \\
\text { and when they are not. }\end{array}$ & [19] \\
\hline $\begin{array}{l}\text { Language } \\
\text { expectancy } \\
\text { theory }\end{array}$ & $\begin{array}{l}\text { It describes that people develop expected } \\
\text { norms about appropriate language usage } \\
\text { in specific contexts. }\end{array}$ & [28] \\
\hline
\end{tabular}




\subsection{Overview of research methods}

Based on our analysis of the literature, quantitative approaches dominate this field of research as they were applied in $77 \%(\mathrm{n}=27)$ of the studies. Only 9\% $(\mathrm{n}=3)$ employed a qualitative approach, and $14 \%(\mathrm{n}$ = 5) combined more than one research method. Overall, the most common method was experiments, followed by surveys, and interviews. Figure 3 presents the research methods used in the identified studies and the number of associated articles.

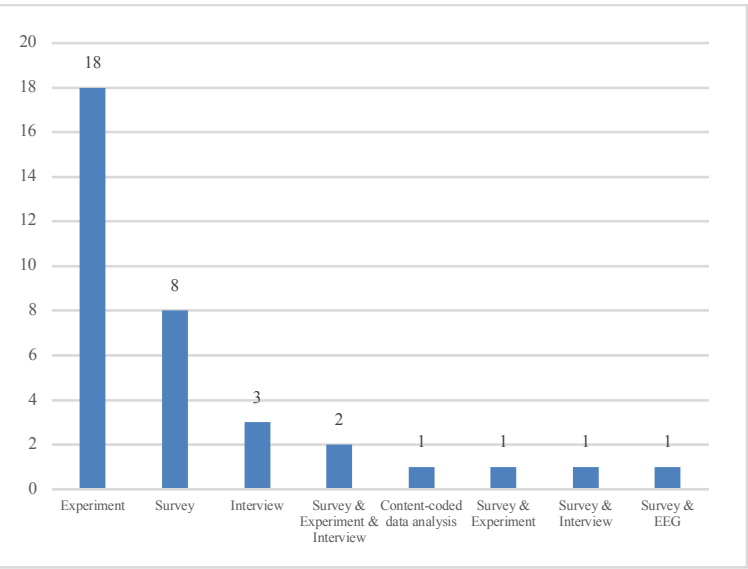

Figure 3. Summary of research methods

\subsection{Overview of research samples}

Our analysis revealed that most studies included students in their samples. Of these, 26\% $(n=9)$ collected data only from students, while $46 \%(n=16)$ used both students and individuals with other demographic profiles in their samples. The researchers argue that incorporating students in samples is important because these individuals are early adopters of emerging technologies [44]. A further $29 \%(n=10)$ of the studies did not provide precise information about their samples' characteristics.

\section{Thematic analysis}

We analyzed the papers using thematic analysis [51], whose overall aim is to capture major themes appeared in the identified articles. Thematic analysis allows researchers to combine an analysis of the frequency of a theme with an analysis of the content as a whole, which allows a broader understanding of the research issue [52].

\subsection{What is anthropomorphism?}

Our literature review indicates that the conceptualization of anthropomorphism in the literature is inconsistent. Researchers have conceptualized anthropomorphism as (1) a tendency, (2) a process, (3) a perception, (4) technological stimuli, and (5) an inference.

Table 2 presents the various definitions of anthropomorphism in the context of AIET. In our sample, about $26 \%(\mathrm{n}=9)$ of the papers conceptualized anthropomorphism as "a tendency" to attribute human or humanlike characteristics to nonhumans. Over one-fifth $(23 \% ; n=8)$ defined it as users' perception of AIET as humanlike. More than one-tenth $(11 \% ; n=4)$ viewed anthropomorphism as technological stimuli that feature human likeness of AIET, such as human appearance, emotions, and motions. About $6 \%(\mathrm{n}=2)$ regarded anthropomorphism as "a process" whereby individuals attribute human or humanlike characteristics to the technology. About $6 \%(\mathrm{n}=2)$ conceptualized anthropomorphism as an inference by which users attribute human likeness to a technology. Finally, 26\% $(\mathrm{n}=9)$ did not provide a definition of anthropomorphism.

The concept of anthropomorphism has been used to capture different aspects of human or humanlike characteristics of AIET, depending on the technological context. For instance, in the context of autonomous vehicles, human characteristics refer to the capacity for rational thought and conscious feeling [21]. In the context of chatbots, Lee et al. [45] viewed human characteristics as users' mental states (e.g., intention and consciousness). In the context of social robots, humanlike characteristics refer to human appearance, which includes psychological (e.g., emotions, personalities, and gestures) and nonpsychological features (e.g., head, eyes, arms, and legs) [22]. In a study of smart speakers, Moussawi et al. [12] described humanlike characteristics as being fluent, respectful, funny, friendly, happy, and caring.

Table 2. Definitions of anthropomorphism

\begin{tabular}{|l|l|l|}
\hline Category & Definition & Reference \\
\hline A tendency & "The tendency to attribute human & {$[13,16$,} \\
& $\begin{array}{l}\text { or humanlike characteristics to } \\
\text { non-human agents" }\end{array}$ & $\begin{array}{l}18-20,33, \\
46]\end{array}$ \\
\cline { 2 - 3 } & $\begin{array}{l}\text { "People tend to associate } \\
\text { humanlike characteristics to these } \\
\text { non-human entities" }\end{array}$ & {$[30]$} \\
\cline { 2 - 3 } & $\begin{array}{l}\text { "People tend to apply their beliefs } \\
\text { and knowledge about humans to } \\
\text { non-human objects when they } \\
\text { have humanlike features" }\end{array}$ & {$[28]$} \\
\hline A process & $\begin{array}{l}\text { "The process by which people } \\
\text { attribute humanlike characteristics } \\
\text { to a non-human entity" }\end{array}$ & {$[26]$} \\
\hline
\end{tabular}




\begin{tabular}{|c|c|c|}
\hline & $\begin{array}{l}\text { "A process of inductive inference } \\
\text { whereby people attribute to } \\
\text { nonhumans distinctively human } \\
\text { characteristics, particularly the } \\
\text { capacity for rational thought } \\
\text { (agency) and conscious feeling } \\
\text { (experience)" }\end{array}$ & {$[21]$} \\
\hline A perception & $\begin{array}{l}\text { "Users' perceptions of AIET as } \\
\text { humanlike" }\end{array}$ & $\begin{array}{l}{[12,15,} \\
24,25,36, \\
37,40, \\
42]\end{array}$ \\
\hline \multirow[t]{2}{*}{$\begin{array}{l}\text { Technological } \\
\text { stimuli }\end{array}$} & $\begin{array}{l}\text { "Humanlike features (e.g., } \\
\text { humanlike appearance, emotions, } \\
\text { personalities, and behaviors) of a } \\
\text { product" }\end{array}$ & {$[3,14,22]$} \\
\hline & $\begin{array}{l}\text { "The extent to which service } \\
\text { robots simulate the characteristics, } \\
\text { behaviors or appearances of } \\
\text { humans" }\end{array}$ & {$[27]$} \\
\hline \multirow[t]{2}{*}{ An inference } & $\begin{array}{l}\text { "Individuals' inferences that a } \\
\text { chatbot's mental states are similar } \\
\text { to those of a human" }\end{array}$ & {$[45]$} \\
\hline & $\begin{array}{l}\text { "Inductive inference in which the } \\
\text { perceiver attributes humanlike } \\
\text { characteristics, motivations, } \\
\text { intentions or underlying mental } \\
\text { states to a non-human entity" }\end{array}$ & [11] \\
\hline Other & $\begin{array}{l}\text { "The assignment of human traits } \\
\text { and characteristics to conversation } \\
\text { agents" }\end{array}$ & {$[38]$} \\
\hline
\end{tabular}

\subsection{How is anthropomorphism measured?}

To understand anthropomorphism in AIET contexts, researchers have employed a variety of measurement techniques. Among the reviewed studies, about $6 \%(\mathrm{n}=2)$ used objective measures of anthropomorphism. By manipulating anthropomorphism conditions in an experimental setting, researchers investigated how either human or cartoonlike images of chatbots or smart speakers influence one's feelings of social and personal interaction with AIET $[33,47]$. Using these objective measures may be useful for comparing the effects of two different anthropomorphism conditions on perceptions of AIET. However, this technique may impede a deeper understanding of peoples' subjective views on or perceptions of the level of anthropomorphism. Therefore, many studies have used subjective measures. Overall, researchers measure anthropomorphism from various perspectives, mainly depending on the type of AIET.

According to Wagner and Schramm-Klein [19], anthropomorphism is a multidimensional construct. However, we found only two studies (6\%) that constructed anthropomorphism with multidimensionally. Araujo [48] constructed anthropomorphism in chatbots with two dimensions: mindful anthropomorphism (i.e., users' perceptions of the technological features of AIET) and mindless anthropomorphism (i.e., the attribution of human likeness to AIET). Wagner et al. [16] proposed three dimensions for conceptualizing anthropomorphism in smart speakers: animacy (i.e., the degree to which users perceive AIET to be lifelike), perceived sociability (i.e., perceived ability of AIET to display sociable behavior), and humanlike fit (i.e., users' attitudes toward human similarity of AIET).

\subsection{What are the antecedents of anthropomorphism?}

We identified several factors that are crucial for evoking anthropomorphism. First, anthropomorphic features have been regarded as a major determinant of anthropomorphism. Cao et al. [9] discerned three types of anthropomorphic features: visual (e.g., appearance, body movement, facial expressions, and gestures), verbal (e.g., voice), and psychological (e.g., autonomy and personality). Indeed, our review showed that visual features, such as humanlike appearance $[28,36$, 40, 41], eyes [13], and emotionality [36]; verbal features, such as voice [21] and humanlike language expressions [34, 48]; and psychological features, such as autonomy of autonomous vehicles [40] can lead to anthropomorphism. Wagner and Schramm-Klein [19] found that social behavior, adaptability, similarity to the user, personality, independence, voice, appearance, and interaction are the factors that contribute to AIET anthropomorphism. However, empirical results are somewhat mixed. Schroeder and Schroeder [39] failed to provide evidence that human voice can induce anthropomorphism and suggested that anthropomorphic features embedded in AIET should reach a threshold of "humanness" to induce anthropomorphism. Second, studies have found that variables related to AIET's performance influence anthropomorphism. For example, Sheehan et al. [11] found that chatbots' performance in communication (i.e., interpreting human utterances and responding to humans with or without errors) can lead to anthropomorphism. Third, the types of relationships with AIET (e.g., AIET as a friend or a servant) can promote anthropomorphism. For example, Kim et al. [17] found that the types of relationships with AIET shape the way in which users experience anthropomorphism. Finally, research has shown that the perceived intelligence of AIET is significantly associated with anthropomorphism $[12,15]$.

\subsection{What are the consequences of anthropomorphism?}

Our analysis shows that anthropomorphism affects the perception, adoption, and continued use of AIET. Some researchers have found that greater 
anthropomorphism in AIET leads to a greater degree of trust [13, 21, 39, 49], likeability [13], perceived warmth and pleasure [17], and AIET morality and dependability of performing tasks [24]. However, other studies have reported contradictory results regarding the consequences of anthropomorphism. For example, Schroeder and Schroeder [39] found that anthropomorphism is negatively associated with trust, whereas Moussawi et al. [12] found no relationship between the two. Kim et al. [36] and Toader et al. [29] reported that there is no relationship between anthropomorphism and the perceived competence of AIET. Similarly, Sah [47] found that anthropomorphism does not affect users' perceptions of AIET.

Our analysis also indicates that anthropomorphism has an influence on AIET adoption. Some studies have found a positive relationship between anthropomorphism and adoption intention [e.g., 11, 26, 45], whereas others have reported a negative relationship $[3, \quad 14, \quad 22]$. Furthermore, anthropomorphism has been suggested to affect AIET adoption through various mechanisms $[3,12,14,16$, 42], such as users' attitudes toward [16], trust in [12, 42], and enjoyment derived from AIET [12].

Finally, our review suggests a positive relationship between anthropomorphism and continued use of AIET [15]. It has also been found that anthropomorphism positively affects individuals' beliefs [34], and purchase intentions [38], increases the likelihood that a user complies with a chatbot's request [20], promotes human-employee rapport building and a positive service experience [27], and has no effect on individuals' perceptions of a company (i.e., attitude toward, emotional connection with, and satisfaction with a company) [48], and individual responses (e.g., service encounter satisfaction, and patronage intention) [29].

\section{Discussion}

\subsection{Recommendations for future research}

Elaborating the concept of anthropomorphism. Our analysis shows that there is still no universally accepted definition and measurement of anthropomorphism. In the reviewed articles, many researchers have conceptualized anthropomorphism as a tendency or a perception (see Table 2). However, after reviewing the operationalizations of anthropomorphism in the AIET context, most research focuses on measuring the level of users' perceptions of AIET as humanlike, without showing a tendency in the measurement. Thus, we suggest that a definition of anthropomorphism in IS field can be the degree to which a user perceives AIET to be humanlike.

Most studies view anthropomorphism as unidimensional, whereas only two of the reviewed studies treat it as a multidimensional concept. Wagner and Schramm-Klein [19] suggested that anthropomorphism as a unidimensional construct may be superficial and that a multidimensional construct can better capture the meaning of anthropomorphism in the context of AIET, as it entails a closer and more detailed consideration. Similarly, Złotowski et al. [50] argued that anthropomorphism as a multidimensional construct can better explain the uncanny valley phenomenon. Accordingly, researchers may benefit from developing a multidimensional scale of anthropomorphism to advance our understanding of anthropomorphism in AIET contexts.

Furthermore, the key term human or humanlike characteristics has been variously defined in terms of the types of AIET or left undefined. Due to this uncertainty in the conceptualization of the term, there is little consensus on the measurement of anthropomorphism. Therefore, it would be meaningful for future studies to clearly and precisely define the term to facilitate more accurate measurements of anthropomorphism and a deeper understanding of its effects on the perception, adoption, and continued use of AIET. Finally, it is important to clearly differentiate between the concept of anthropomorphism and other similar concepts (e.g., perceived humanness, perceived intelligence, and anthropomorphic cues). By clearly framing the concept of anthropomorphism, the phenomenon, its antecedents, and its consequences can be better comprehended.

Identifying the antecedents of anthropomorphism. We identified a wide range of variables related to anthropomorphism. Importantly, the various types of anthropomorphic features have not received equal attention. Studies on visual features, especially humanlike appearance, dominate the relevant research, while verbal and psychological features remain underexplored. Except humanlike appearance and language expressions, humanlike eyes, voices, behaviors, and autonomy of AIET are also underexplored (represented by only one study in our sample), which limits our understanding of the role of the various types of anthropomorphic features in inducing anthropomorphism. Future research should examine how a type of anthropomorphic feature or a combination of types influences the level of anthropomorphism. For example, for smart speakers such as Amazon Alexa, researchers may focus on verbal and psychological features, as Alexa currently lacks any obvious visual features. In social robots, 
visual, verbal, and psychological features could be examined simultaneously.

Understanding the consequences of anthropomorphism. Our findings suggest that anthropomorphism plays a positive role in shaping the perception and adoption intention of AIET. However, we also found that anthropomorphism has insignificant or negative effects on perceptions and adoption intention. Despite the increased scholarly attention to the role of anthropomorphism in AIET, relatively few studies have explained how and why anthropomorphism exerts insignificant or negative effects. Therefore, the underlying mechanisms should be further explored. Moreover, it may be useful to examine whether these insignificant or negative effects are produced by an improper combination of anthropomorphic features embedded in AIET. Furthermore, it may be beneficial to investigate these effects from the perspectives of the uncanny valley, expectancy violations, and mental-model differences.

Reconsidering the theoretical foundation. Our analysis shows that although various theories have been applied to explain anthropomorphism in the AIET context (see Table 1), many studies ( $31 \%$ of our sample) lack theoretical foundations. This may be an obstacle to improving our understanding of the phenomenon of anthropomorphism in the context of AIET. Thus, it would be meaningful to employ theories and frameworks in future studies. Moreover, given that anthropomorphism in AIET has opened a wide research area for IS researchers, existing theories may not be sufficient to explain the phenomenon accurately and comprehensively. One possible direction for future research is to use qualitative methods to provide new insights that may help to develop new theories. Existing and new theories may work complementarily in the effort to elucidate the phenomenon of anthropomorphism in AIET.

Diversifying research methods to capture anthropomorphism. Our review shows that experiments and surveys are the dominant research methods. Other approaches, such as qualitative (e.g., interviews and case studies) and mixed methods, are less frequently used. Future studies should diversify the research methods to examine the effects of anthropomorphism in AIET from multiple perspectives. Furthermore, given that anthropomorphism can have insignificant or negative effects on the perception and adoption intention of AIET, triangulation could be used to collect both subjective and objective data, thus enabling more comprehensive analyses.

\subsection{Theoretical implications}

This study contributes to the literature in several ways. First, we provide an overview of the current state of research, offering researchers an overall view of the phenomenon and related research issues. Second, our overview of the existing definitions and measurements of anthropomorphism can provide a better understanding of anthropomorphism in various types of AIET. Third, we identify and summarize a wide range of factors contributing to anthropomorphism, as well as its consequences, providing deeper insights into how to induce anthropomorphism and understand its results. Finally, we identify research gaps and suggest directions for future research. Our suggestions may help researchers better uncover the phenomenon of anthropomorphism, explore its antecedents and consequences in future studies.

\subsection{Practical implications}

Our study also has practical implications for AIET developers. We highlight the three types of anthropomorphic features (i.e., visual, verbal, and psychological) that can induce anthropomorphism. Based on these, AIET developers may consider enriching the anthropomorphic features of AIET according to its different types. For example, people could use voice to interact with chatbots or control autonomous vehicles in the future. Importantly, we found that anthropomorphism does not always play a positive role in AIET adoption intention. To increase acceptance of the technology, AIET developers should identify and rectify the conditions under which anthropomorphism may exert an insignificant or negative effect on adoption intention.

\subsection{Limitations}

This research has several limitations. First, we selected the papers based on the inclusion and exclusion criteria. Therefore, there might be some important and relevant papers that have been omitted. For example, non-empirical studies from the academic, industry reports and magazines. Second, our research focuses on the more recent research on AIET and anthropomorphism. Based on the inclusion and exclusion criteria, we reviewed a limited number of empirical studies $(\mathrm{n}=35)$. Thus, it is not sufficient for us to conduct a quantitative meta-analysis in the current study. Future studies may be performed a meta-analysis to improve our understanding of the interplay between anthropomorphism, its antecedents and consequences. 


\section{References}

[1] R.E. Bawack, S. Fosso Wamba, and K.D.A. Carillo. Artificial intelligence in practice: Implications for IS research. in 25th Americas Conference on Information Systems (AMCIS). 2019. Cancún, Mexico: AIS.

[2] S.A. Alsheibani, D. Cheung, and D. Messom. Factors inhibiting the adoption of artificial intelligence at organizational-level: A preliminary investigation. in 25th Americas Conference on Information Systems (AMCIS). 2019. Cancún, Mexico: AIS.

[3] H. Lin, O.H. Chi, and D. Gursoy, "Antecedents of customers' acceptance of artificially intelligent robotic device use in hospitality services", Journal of Hospitality Marketing \& Management, 2019: pp. 1-20.

[4] K.-H. Yu, A.L. Beam, and I.S. Kohane, "Artificial intelligence in healthcare", Nature Biomedical Engineering 2018, 2(10): pp. 719-731.

[5] K. Jha, A. Doshi, P. Patel, and M. Shah, "A comprehensive review on automation in agriculture using artificial intelligence", Artificial Intelligence in Agriculture, 2019, 2: pp. 1-12.

[6] M. Chassignol, A. Khoroshavin, A. Klimova, and A. Bilyatdinova, "Artificial intelligence trends in education: A narrative overview", Procedia Computer Science, 2018, 136: pp. 16-24.

[7] L. Rouhiainen, Artificial intelligence: 101 things you must know today about our future. Lasse Rouhiainen,2018.

[8] F.B. Insights, Market research report. 2020.

[9] C. Cao, L. Zhao, and Y. Hu. Anthropomorphism of intelligent personal assistants (IPAs): Antecedents and consequences. in 23rd Pacific Asia Conference on Information Systems (PACIS). 2019. Xi'an, China: AIS.

[10] C. Rzepka and B. Berger. User interaction with AIenabled systems: A systematic review of IS research. in 29th International Conference on Information Systems (ICIS). 2018. San Francisco, CA, the United States: AIS.

[11] B. Sheehan, H.S. Jin, and U. Gottlieb, "Customer service chatbots: Anthropomorphism and adoption", Journal of Business Research, 2020, 115: pp. 14-24.

[12] S. Moussawi, M. Koufaris, and R. Benbunan-Fich, "How perceptions of intelligence and anthropomorphism affect adoption of personal intelligent agents", Electronic Markets, 2020: pp. 1-22.

[13] D. Niu, J. Terken, and B. Eggen, "Anthropomorphizing information to enhance trust in autonomous vehicles", Human Factors and Ergonomics in Manufacturing \& Service Industries, 2018, 28(6): pp. 352-359.

[14] D. Gursoy, O.H. Chi, L. Lu, and R. Nunkoo, "Consumers acceptance of artificially intelligent (AI) device use in service delivery", International Journal of Information Management, 2019, 49: pp. 157-169.

[15] S. Moussawi and M. Koufaris. Perceived intelligence and perceived anthropomorphism of personal intelligent agents: Scale development and validation. in 52nd Hawaii International Conference on System Sciences (HICSS). 2019. Grand Wailea, Maui, the United States: University of Hawaii at Manoa.

[16] K. Wagner, F. Nimmermann, and H. Schramm-Klein. Is it human? The role of anthropomorphism as a driver for the successful acceptance of digital voice assistants. in 52nd
Hawaii International Conference on System Sciences (HICSS). 2019. Grand Wailea, Maui, the United States: University of Hawaii at Manoa.

[17] A. Kim, M. Cho, J. Ahn, and Y. Sung, "Effects of gender and relationship type on the response to artificial intelligence", Cyberpsychology, Behavior, and Social Networking, 2019, 22(4): pp. 249-253.

[18] F. Schweitzer, R. Belk, W. Jordan, and M. Ortner, "Servant, friend or master? The relationships users build with voice-controlled smart devices", Journal of Marketing Management, 2019, 35(7-8): pp. 693-715.

[19] K. Wagner and H. Schramm-Klein. Alexa, are you human? Investigating anthropomorphism of digital voice assistants-a qualitative approach. in 50th International Conference on Information Systems (ICIS). 2019. Munich, Germany: AIS.

[20] M. Adam, M. Wessel, and A. Benlian, "AI-based chatbots in customer service and their effects on user compliance", Electronic Markets 2020: pp. 1-19.

[21] A. Waytz, J. Heafner, and N. Epley, "The mind in the machine: Anthropomorphism increases trust in an autonomous vehicle", Journal of Experimental Social Psychology, 2014, 52: pp. 113-117.

[22] L. Lu, R. Cai, and D. Gursoy, "Developing and validating a service robot integration willingness scale", International Journal of Hospitality Management, 2019, 80: pp. 36-51.

[23] J. Webster and R.T. Watson, "Analyzing the past to prepare for the future: Writing a literature review", MIS Quarterly, 2002, 26(2): pp. xiii-xxiii.

[24] J. Banks, "A perceived moral agency scale: Development and validation of a metric for humans and social machines", Computers in Human Behavior, 2019, 90: pp. 363-371.

[25] C. Rzepka, B. Berger, and T. Hess. Why another customer channel? Consumers' perceived benefits and costs of voice commerce. in 53rd Hawaii International Conference on System Sciences (HICSS). 2020. Maui, Hawaii, the United States: University of Hawaii at Manoa. [26] S. Melián-González, D. Gutiérrez-Taño, and J. Bulchand-Gidumal, "Predicting the intentions to use chatbots for travel and tourism", Current Issues in Tourism, 2019: pp. 1-19.

[27] H. Qiu, M. Li, B. Shu, and B. Bai, "Enhancing hospitality experience with service robots: The mediating role of rapport building", Journal of Hospitality Marketing \& Management, 2020, 29(3): pp. 247-268.

[28] S. Choi, S.Q. Liu, and A.S. Mattila, "'How may i help you?" says a robot: Examining language styles in the service encounter", International Journal of Hospitality Management, 2019, 82: pp. 32-38.

[29] D.-C. Toader, G. Boca, R. Toader, M. Măcelaru, C. Toader, D. Ighian, and A.T. Rădulescu, "The effect of social presence and chatbot errors on trust", Sustainability, 2020, 12(1): pp. 1-24.

[30] I. Seeber, E. Bittner, R.O. Briggs, T. de Vreede, G.-J. de Vreede, A. Elkins, R. Maier, A.B. Merz, S. Oeste-Reiß, and N. Randrup, "Machines as teammates: A research agenda on AI in team collaboration", Information \& Management, 2020, 57(2): pp. 1-22. 
[31] Y. Moon, "Intimate exchanges: Using computers to elicit self-disclosure from consumers", Journal of Consumer Research, 2000, 26(4): pp. 323-339.

[32] C. Nass and Y. Moon, "Machines and mindlessness: Social responses to computers", Journal of Social Issues, 2000, 56(1): pp. 81-103.

[33] T. Verhagen, J. Van Nes, F. Feldberg, and W. Van Dolen, "Virtual customer service agents: Using social presence and personalization to shape online service encounters", Journal of Computer-Mediated Communication, 2014, 19(3): pp. 529-545.

[34] S. Diederich, S. Lichtenberg, A.B. Brendel, and S. Trang. Promoting sustainable mobility beliefs with persuasive and anthropomorphic design: Insights from an experiment with a conversational agent. in 40th International Conference on Information Systems (ICIS). 2019. Munich, Germany: AIS.

[35] S. Diederich, A.B. Brendel, S. Lichtenberg, and L. Kolbe. Design for fast request fulfillment or natural interaction? Insights from an experiment with a conversational agent. in 27th European Conference on Information Systems (ECIS). 2019. Stockholm-Uppsala, Sweden: AIS.

[36] S.Y. Kim, B.H. Schmitt, and N.M. Thalmann, "Eliza in the uncanny valley: Anthropomorphizing consumer robots increases their perceived warmth but decreases liking", Marketing Letters, 2019, 30(1): pp. 1-12.

[37] C.-E. Yu, "Humanlike robots as employees in the hotel industry: Thematic content analysis of online reviews", Journal of Hospitality Marketing \& Management, 2020, 29(1): pp. 22-38.

[38] C. Yen and M.-C. Chiang, "Trust me, if you can: A study on the factors that influence consumers' purchase intention triggered by chatbots based on brain image evidence and self-reported assessments", Behaviour \& Information Technology, 2020: pp. 1-18.

[39] J. Schroeder and M. Schroeder. Trusting in machines: How mode of interaction affects willingness to share personal information with machines. in 51st Hawaii International Conference on System Sciences (HICSS). 2018. Hilton Waikoloa Village, Hawaii: University of Hawaii at Manoa.

[40] J.-G. Lee, K.J. Kim, S. Lee, and D.-H. Shin, "Can autonomous vehicles be safe and trustworthy? Effects of appearance and autonomy of unmanned driving systems", International Journal of Human-Computer Interaction, 2015, 31(10): pp. 682-691.

[41] E. Go and S.S. Sundar, "Humanizing chatbots: The effects of visual, identity and conversational cues on humanness perceptions", Computers in Human Behavior, 2019, 97: pp. 304-316.

[42] M. Bruckes, J.-G. Grotenhermen, F. Cramer, and G. Schewe. Paving the way for adoption of autonomous driving: Institution-based trust as a critical success factor. in 27th European Conference on Information Systems (ECIS). 2019. Stockholm-Uppsala, Sweden: AIS.

[43] Orbisresearch, Artificial intelligence (AI) market share, growth by top company, geographical region, application, driver, trends \& forecast to 2027. 2020.

[44] X. Luo, H. Li, J. Zhang, and J.P. Shim, "Examining multi-dimensional trust and multi-faceted risk in initial acceptance of emerging technologies: An empirical study of mobile banking services", Decision Support Systems, 2010, 49(2): pp. 222-234.

[45] S. Lee, N. Lee, and Y.J. Sah, "Perceiving a mind in a chatbot: Effect of mind perception and social cues on copresence, closeness, and intention to use", International Journal of Human-Computer Interaction, 2020, 36(10): pp. 930-940.

[46] C. Oh, T. Lee, Y. Kim, S. Park, S. Kwon, and B. Suh. Us vs. them: Understanding artificial intelligence technophobia over the google deepmind challenge match. in 2017 CHI Conference on Human Factors in Computing Systems (CHI). 2017. Denver, CO, the United States: ACM. [47] Y.J. Sah, "Talking to a pedagogical agent in a smart TV: Modality matching effect in human-TV interaction", Behaviour \& Information Technology, 2019: pp. 1-11.

[48] T. Araujo, "Living up to the chatbot hype: The influence of anthropomorphic design cues and communicative agency framing on conversational agent and company perceptions", Computers in Human Behavior, 2018, 85: pp. 183-189.

[49] N. Mesbah, C. Tauchert, C.M. Olt, and P. Buxmann. Promoting trust in AI-based expert systems. in 25th Americas Conference on Information Systems (AMCIS). 2019. Cancun, Mexico: AIS.

[50] J. Złotowski, E. Strasser, and C. Bartneck. Dimensions of anthropomorphism: from humanness to humanlikeness. in 9th ACM/IEEE International Conference on Human-Robot Interaction (HRI). 2014. Bielefeld, Germany: IEEE.

[51] V. Braun, V. Clarke. "Using thematic analysis in psychology", Qualitative Research in Psychology, 2006, 3 (2): pp. 77-101.

[52] M. I. Alhojailan. "Thematic analysis: A critical review of its process and evaluation", West East Journal of Social Sciences, 2012, 1(1): pp. 39-47. 